\title{
Article \\ Precanonical Structure of the Schrödinger Wave Functional of a Quantum Scalar Field in Curved Space-Time
}

\author{
Igor V. Kanatchikov ${ }^{1,2}$ \\ 1 School of Physics and Astronomy, University of St Andrews, St Andrews KY16 9SS, UK; \\ ik25@st-andrews.ac.uk \\ 2 National Quantum Information Center in Gdańsk (KCIK), 81-831 Sopot, Poland; kai@fuw.edu.pl
}

\begin{abstract}
A relationship between the functional Schrödinger representation and the precanonical quantization of a nonlinear scalar field theory is extended to arbitrary curved space-times. The canonical functional derivative Schrödinger equation is derived from the manifestly covariant precanonical Schrödinger equation in a singular limiting case when the ultraviolet parameter $\varkappa$ introduced by precanonical quantization is identified with the invariant delta-function at equal spatial points. In the same limiting case, the Schrödinger wave functional is expressed as the trace of the multidimensional product integral of Clifford-algebra-valued precanonical wave functions restricted to a certain field configuration. Thus the standard QFT in curved space-time in functional Schrödinger representation emerges from the precanonical formulation of quantum fields as a singular limiting case.
\end{abstract}

Keywords: quantum field theory in curved space-time; De Donder-Weyl Hamiltonian formalism; precanonical quantization; canonical quantization; functional Schrödinger representation

PACS: 03.70.+k, 04.62.+v, 11.90.+t, 02.90.+p

\section{Introduction}

Since the theoretical discovery of the Hawking radiation of black holes, quantum field theory in curved space-time [1] often has been considered as an opportunity to study an interplay between gravitation, space-time and quantum theory in order to gain insights and intuitions into the quantum geometry of space-time and the quantum theory of gravity. The consideration of quantum fields on curved backgrounds also allows us to understand what concepts and mathematical structures are important in quantum field theory beyond the simplifying framework of the Poincaré-invariant Minkowski spacetime.

Recently we have put forward an approach to quantization of fields called precanonical quantization [2-5] which is based on mathematical structures of the De Donder-Weyl (DW) Hamiltonian theory known in the calculus of variations [6]. This Hamiltonian-like formulation does not require a space-time decomposition and treats all space-time variables on equal footing. In this sense it is an intermediate description of classical fields between the Lagrangian one and the canonical Hamiltonian one (hence the name "precanonical") which allows us to avoid the necessity of treating fields as infinite-dimensional Hamiltonian systems at least on the level of formulating the corresponding quantum theory of fields and hence circumvent technical difficulties the canonical quantization of fields brings in. 
In DW Hamiltonian theory a proper generalization of Poisson brackets is defined on the dynamical variables represented by differential forms rather than functionals. The brackets are defined by the structure we called polysymplectic and they lead to the structure of Poisson-Gerstenhaber algebra $[5,7,8]$ (see also [9-14] for further generalizations). The latter generalizes the Poisson algebra in the canonical Hamiltonian formalism, which underlines canonical quantization and hence underlines a different procedure of quantization of fields which we call precanonical quantization.

The DW Hamiltonian formulation is related to a generalization of the Hamilton-Jacobi (HJ) theory which is formulated in terms of partial derivative rather than functional derivative equations [6]. Precanonical quantization clarifies the question as to which formulation of quantum theory of fields reproduces the DWHJ equation in the classical limit $[3,4]$. We found that quantization of a small Heisenberg-like subalgebra of the aforementioned Poisson-Gerstenhaber algebra leads to a hypercomplex generalization of the formalism of quantum theory where both operators and wave functions are Clifford-algebra-valued. The precanonical analogue of the Schrödinger equation is formulated using the Dirac operator on the space-time which appears as a multidimensional generalization of the time derivative in the left side of the standard Schrödinger equation [2-5].

One of the features of precanonical formulation of quantized fields is that it allows us to reproduce the classical field equations in DW Hamiltonian form as the equations for expectation values of operators defined by precanonical quantization and evolving according to the precanonical Schrödinger equation $[15,16]$. By treating the space-time variables on equal footing it also leads to a formulation of quantum theory of fields on a finite-dimensional space of field and space-time variables thus providing a new framework for the quantum gauge theory [31,34] and the theory of quantum gravity $[33,35]$ which looks more promising both conceptually and from the point of view of a posibility of rigorous mathematical treatment.

An important aspect of realization of the potential of precanonical quantization is understanding of how it could be related to more familiar and already working concepts of standard QFT. In this paper, we extend our previous results on the relationship between precanonical quantization and the functional Schrödinger picture in QFT [27-32] to scalar field theory on arbitrary curved space-times.

We proceed as follows. In Section 2 we first remind the results of canonical quantization of scalar field theory on curved space-time in the functional Schrödinger representation and precanonical quantization of the same system, and then we discuss drastic differences between them. Section 2 also serves to introduce the notations used throughout the paper. The connection between the functional Schrödinger representation and the results of precanonical quantization in curved space-time is established in Section 3 which consists of several subsections reflecting the multi-step nature of the argument. Namely, we first outline a general idea which allows us to anticipate a connection between the Schrödinger wave functional and precanonical wave function based on the respective probabilistic interpretation of both objects. Second, in sub-section 3.1, we present a restriction of precanonical Schödinger equation to the section of the bundle of field coordinates over space-time, which represents a field configuration the Schrödinger wave functional is a functional of. The restriction of precanonical Schrödinger equation is formulated in terms of the total covariant derivative introduced in sub-section 3.1.1 and it allows us to write in sub-section 3.2 the equation for the time evolution of the wave functional composed from precanonical wave functions. To proceed with the derivation of the Schödinger equation for the wave functional from the restriction of the precanonical Schrödinger equation to a field configuration, in sub-section 3.3 we evaluate the functional derivatives of the functional composed from precanonical wave function with respect to the field variables. Then, in Section 4, we analyze different terms in the equation presented in sub-section 3.2 and show how they can be expressed in terms of the functional derivatives of the composed functional or cancelled in a certain limiting case. The result of this section is the derivation of the functional derivative Schrödinger equation from the restricted precanonical Schrödinger equation up to an additional term which involves the commutator of the zeroth component of the spin connection matrix with the precanonical wave function, see eq. (37). In Section 4 we consider static space-times with the vanishing 
zeroth component of the spin connection and obtain the expression of the Schrödinger wave functional as the trace of the continual product or product integral of precanonical wave functions restricted to a field configuration. A more general case of non-static space-times with non-vanishing zeroth component of the spin connection is considered in Section 5 where we show that the extraneous term in (37) with the commutator of the zeroth component of spin connection with precanonical wave function disappears if the wave functional is expressed in terms of transformed precanonical wave functions with the transformation given by the time-ordered exponential of the zeroth component of spin connection. This observation allows us to extend the results from the static space-times to nonstatic ones. In Section 6 we present our conclusions and highlight the main steps of the derivation of the functional Schrödinger equation from precanonical Schödinger equation and the expression of the Schrödinger wave functional as a product integral of precanonical wave functions or their transforms. We also discuss the physical meaning of the ultra-violet parameter $\varkappa$ whose infinite value corresponds to the limiting case in which it is shown to be possible to derive the standard functional Schrödinger representation of QFT from precanonical quantization.

\section{Quantum scalar field on a curved space-time: the canonical and precanonical descriptions}

Let us recall that the conventional canonical quantization of scalar field theory in curved space-time can be formulated in the functional Schrödinger representation of QFT [24,25]. It leads [17-23]) to the description of the corresponding quantum field in terms of the Schrödinger wave functional $\Psi([\phi(\mathbf{x})], t)$ satisfying the Schrödinger equation

$$
\mathrm{i} \hbar_{t} \Psi=\int d \mathbf{x} \sqrt{g}\left(\frac{\hbar^{2}}{2} \frac{g_{00}}{g} \frac{\delta^{2}}{\delta \phi(\mathbf{x})^{2}}-\frac{1}{2} g^{i j} \partial_{i} \phi(\mathbf{x}) \partial_{j} \phi(\mathbf{x})+V(\phi)\right) \Psi,
$$

where the right hand side is the canonical Hamiltonian operator formulated in terms of functional derivative operators, $x^{\mu}=(t, \mathbf{x})=\left(t, x^{i}\right)$ are space-time coordinates, $g_{\mu v}$ is the space-time metric tensor whose components depend on $x^{\mu}, g=\left|\operatorname{det}\left(g_{\mu \nu}\right)\right|$. In (1) one uses the space-time coordinates adapted to the space-like foliation such as the induced metric on the space-like leaves of the foliation is $g_{i j}$, the lapse $N=\sqrt{g_{00}}$ and the shift functions $N_{i}=g_{0 i}=0$.

The precanonical quantization of a scalar field $\phi(x)$ on a curved space-time background given by the metric tensor $g_{\mu v}(x)$ (cf. $\left.[15,16]\right)$ gives rise to the description in terms of a wave function $\Psi\left(\phi, x^{\mu}\right)$ on the finite-dimensional bundle with the coordinates $\left(\phi, x^{\mu}\right)$ which takes values in the complexified space-time Clifford algebra, i.e.

$$
\Psi=\psi+\psi_{\mu} \gamma^{\mu}+\frac{1}{2 !} \psi_{\mu_{1} \mu_{2}} \gamma^{\mu_{1} \mu_{2}}+\ldots+\frac{1}{n !} \psi_{\mu_{1} \ldots \mu_{n}} \gamma^{\mu_{1} \ldots \mu_{n}},
$$

and satisfies the partial derivative precanonical Schrödinger equation (pSE)

$$
\mathrm{i} \hbar \gamma^{\mu}(x) \nabla_{\mu} \Psi=\left(-\frac{1}{2} \hbar^{2} \varkappa \frac{\partial^{2}}{\partial \phi^{2}}+\frac{1}{\varkappa} V(\phi)\right) \Psi=: \frac{1}{\varkappa} \widehat{H} \Psi,
$$

where $\gamma^{\mu}(x)$ are the curved space-time Dirac matrices such that

$$
\gamma^{\mu}(x) \gamma^{\nu}(x)+\gamma^{\nu}(x) \gamma^{\mu}(x)=2 g^{\mu \nu}(x),
$$

$\gamma^{\mu_{1} \ldots \mu_{p}}$ are the antisymmetrized products of $p$ Dirac matrices,

$$
\nabla_{\mu}:=\partial_{\mu}+\omega_{\mu}(x)
$$


is the covariant derivative with the spin-connection matrices $\omega_{\mu}(x)=\frac{1}{4} \omega_{\mu A B}(x) \underline{\gamma}^{A B}$ (see e.g. [26]) acting on Clifford-algebra-valued wave functions by the commutator product [32], and $\gamma^{A}$ are the constant Dirac matrices which factorize the Minkowski metric $\eta^{A B}$ of the tangent space ${ }^{1}$ :

$$
\underline{\gamma}^{A} \underline{\gamma}^{B}+\underline{\gamma}^{B} \underline{\gamma}^{A}=2 \eta^{A B}
$$

The operator $\widehat{H}$ in (2) is the De Donder-Weyl (DW) Hamiltonian operator constructed according to the procedure of precanonical quantization $[3,4,15,16]$. In the expression of $\hat{H}$ there appears an ultraviolet parameter $\varkappa$ of the dimension of the inverse spatial volume. This parameter typically appears in the representations of precanonical quantum operators [2-4,15]. For the scalar fields on curved background the DW Hamiltonian operator $\widehat{H}$ coincides with its couterpart in flat space-time (cf. $[2-4,15])$. Correspondingly, the curved space-time manifests itself only through the curved space-time Dirac matrices (3) and the spin-connection in the left-hand side of (2).

As we have seen, the description of quantum fields obtained from precanonical quantization is very different from a familiar description of quantum fields derived from the canonical quantization. In particular, while in the description using the functional Schrödinger picture the role of space variables $\mathbf{x}$ is different from the role the time variable $t$, the precanonical description is entirely space-time symmetric, manifestly covariant and independent of the assumption of global hyperbolicity of space-time. One can also wonder how the description in terms of precanonical wave function on a finite-dimensional space and the corresponding partial derivative precanonical Schrödinger equation can match the description in terms of functionals on an infinite-dimensional space of field configurations at a fixed time and the corresponding functional derivative Schrödinger equation, or how the multiparticle states and multi-point correlation functions of standard QFT could be related to the natural objects within the precanonical description such as the Green function of the precanonical Schrödinger equation (2).

However, one can reduce the perceived gap between those two descriptions by noticing that already on the classical level the solutions of field equations can be equally well treated using both the language of partial derivative equation on a finite dimensional space (in the Lagrangian, DW Hamiltonian and DWHJ descriptions) and the language of functional derivative equations (in the canonical Hamiltonian and Hamilton-Jacobi description). Moreover, one can derive the canonical Hamiltonian and HJ equations from the DW Hamiltonian and DWHJ equations, respectively (see e.g. $[27,30])$. In the next section, we will show how those relationships between the canonical and precanonical are extended to the quantum level in curved space-times.

\section{Relating the precanonical wave function and the Schrödinger wave functional}

Our preceding work has established a relationship between the functional Schrödinger representation and precanonical quantization of scalar and Yang-Mills in flat space-time [29-31]. The familiar QFT in functional Schrödinger representation was derived from the precanonical quantization as the limiting case when the combination $\gamma_{0} \varkappa$ is replaced by $\delta(\mathbf{0})$, a regularized value of Dirac delta function $\delta\left(\mathbf{x}-\mathbf{x}^{\prime}\right)$ at coinciding spatial points, which can be understood as the cutoff of the momentum space volume introduced by a regularization. Here we intend to extend this relationship to curved space-time using the example of a quantum scalar field.

The Schrödinger wave functional $\mathbf{\Psi}([\phi(\mathbf{x})], t)$ is interpreted as the probability amplitude of finding a field configuration $\phi(\mathbf{x})$ at some moment of time $t$. The precanonical wave function

1 We chose the signature $+--\ldots$. Note that in this paper we depart from our notation in [16] where the flat Dirac matrices were denoted $\bar{\gamma}^{A}$. This notation can be confusing when the Dirac conjugate matrix has to be denoted as $\bar{\gamma}^{A}$. As for the rest, throughout this paper we mostly follow the notation and conventions used in $[15,16,29,30]$. In particular, the plane capital Greek letters like $\Psi$ and $\Phi$ denote wave functions on a finite dimensional space of $\phi$ and $x^{\mu}$ and the boldface capital Greek letters like $\boldsymbol{\Psi}$ and $\boldsymbol{\Phi}$ denote functionals of field configurations $\phi(\mathbf{x})$. 
$\Phi(\phi, x)$ is the probability amplitude of observing the field value $\phi$ at the space-time point $x$. Then the time-dependent complex functional probability amplitude $\Psi([\phi(\mathbf{x})], t)$ can be expected to be a composition of space-time dependent Clifford-valued probability amplitudes given by the precanonical wave function $\Psi(\phi, x)$. It means that the Schrödinger wave functional $\Psi([\phi(\mathbf{x})], t)$ is a functional of precanonical wave functions $\Psi(\phi, x)$ restricted to a specific field configuration which is represented by a section $\Sigma$ in the total space of the bundle with the coordinates $(\phi, x)$, which is defined by the equation $\Sigma: \phi=\phi(\mathbf{x})$ at time $t$. Thus by denoting the restriction of precanonical wave function $\Psi(\phi, x)$ to $\Sigma$ as

$$
\Psi_{\Sigma}(\mathbf{x}, t):=\Psi(\phi=\phi(\mathbf{x}), \mathbf{x}, t)
$$

we assume that

$$
\mathbf{\Psi}([\phi(\mathbf{x})], t)=\mathbf{\Psi}\left(\left[\Psi_{\Sigma}(\mathbf{x}, t), \phi(\mathbf{x})\right]\right),
$$

so that the time dependence of the wave functional $\Psi$ is totally controlled by the time dependence of precanonical wave function restricted to $\Sigma$. Then the chain rule differentiation yields the time derivative of $\Psi$

$$
\mathrm{i} \partial_{t} \Psi=\operatorname{Tr} \int d \mathbf{x}\left\{\frac{\delta \Psi}{\delta \Psi_{\Sigma}^{T}(\mathbf{x}, t)} \mathrm{i} \partial_{t} \Psi_{\Sigma}(\mathbf{x}, t)\right\}
$$

\subsubsection{Total covariant derivative}

Let us introduce the notion of the total covariant derivative acting on Clifford-algebra-valued tensors, particularly on those restricted to $\Sigma$. The derivative will be called "total" in the sense that (i) when acting on a Clifford-valued tensor function $T_{\nu_{1} v_{2} \ldots}^{\mu_{1} \mu_{2} \ldots}$ it includes both the spin-connection matrix $\omega_{\mu}$ and the Christoffel symbols $\Gamma_{\beta \gamma}^{\alpha}$ (c.f. [36]) and (ii) when a tensor quantity with the components 
depending both on $x$ and $\phi$ is restricted to $\Sigma$, its derivative with respect to $x$-s is understood in the sense of the total derivative (9):

$$
\begin{aligned}
\nabla_{\alpha}^{\operatorname{tot}} T_{v_{1} \nu_{2} \ldots}^{\mu_{1} \mu_{2} \ldots}:= & \frac{d}{d x^{\alpha}} T_{v_{1} v_{2} \ldots}^{\mu_{1} \mu_{2} \ldots}+\left[\omega_{\alpha}, T_{v_{1} \nu_{2} \ldots}^{\mu_{1} \mu_{2} \ldots}\right] \\
& +\Gamma_{\alpha \beta}^{\mu_{1}} T_{v_{1} v_{2} \ldots}^{\beta \mu_{2} \ldots}+\Gamma_{\alpha \beta}^{\mu_{2}} T_{v_{1} v_{2} \ldots}^{\mu_{1} \nu_{2} \ldots}+\ldots \\
& -\Gamma_{\alpha v_{1}}^{\beta} T_{\beta v_{2} \ldots}^{\mu_{1} \mu_{2} \ldots}-\Gamma_{\alpha v_{2}}^{\beta} T_{v_{1} \beta_{1} \ldots}^{\mu_{1} \mu_{2} \ldots}-\ldots
\end{aligned}
$$

The commutator in the second term guarantees that the total covariant derivative of the Clifford product of two Clifford-valued tensor quantities fulfills the Leibniz rule. The Christoffel symbols appear in the covariant derivative of non-scalar Clifford quantities, e.g. in the condition of metric compatibility

$$
\nabla_{\alpha}^{\text {tot }} \gamma^{\mu}=0,
$$

where only the first partial derivative term in (9) is non-vanishing when acting on $x$-dependent $\gamma$-matrices.

Now, in terms of the total covariant derivative $\nabla^{\text {tot }}$ acting on $\Psi_{\Sigma}$ eq. (8) takes the form

$$
\mathrm{i} \partial_{t} \Psi_{\Sigma}=-\mathrm{i} \gamma_{0} \gamma^{i} \nabla_{i}^{\mathrm{tot}} \Psi_{\Sigma}-\mathrm{i}\left[\omega_{0}, \Psi_{\Sigma}\right]+\mathrm{i} \gamma_{0} \gamma^{i} \partial_{i} \phi(\mathbf{x}) \partial_{\phi} \Psi_{\Sigma}+\frac{1}{\varkappa} \gamma_{0} \widehat{H}_{\Sigma} \Psi_{\Sigma} .
$$

\subsection{Time evolution of the Schrödinger wave functional from $p S E$}

From (7), (8) and (10) the equation of the time evolution of the wave functional (6) constructed from precanonical wave functions takes the form

$$
\begin{aligned}
i \partial_{t} \boldsymbol{\Psi}= & \operatorname{Tr} \int \mathrm{d} \mathbf{x}\left\{\frac{\delta \boldsymbol{\Psi}}{\delta \Psi_{\Sigma}^{T}(\mathbf{x}, t)}[\underbrace{-\mathrm{i} \gamma_{0} \gamma^{i} \frac{d}{d x^{i}} \Psi_{\Sigma}(\mathbf{x})}_{I}+\underbrace{\mathrm{i} \gamma_{0} \gamma^{i} \partial_{i} \phi(\mathbf{x}) \partial_{\phi} \Psi_{\Sigma}(\mathbf{x})}_{I I}\right. \\
& \underbrace{-\frac{\mathrm{i}}{4} \gamma_{0} \gamma^{i}\left[\omega_{i}, \Psi_{\Sigma}(\mathbf{x})\right]}_{I I I a} \underbrace{-\frac{\mathrm{i}}{4}\left[\omega_{0}, \Psi_{\Sigma}\right]}_{I I I b} \underbrace{-\frac{\varkappa}{2} \gamma_{0} \partial_{\phi \phi} \Psi_{\Sigma}(\mathbf{x})}_{I V}+\underbrace{\frac{1}{\varkappa} \gamma_{0} V(\phi(\mathbf{x})) \Psi_{\Sigma}(\mathbf{x})}_{V}]\} .
\end{aligned}
$$

In order to derive from this equation the functional derivative Schrödinger equation (1) we need to try to express the terms in the right hand side of (14) in terms of the functional derivatives $f$ the composite functional $\Psi$ in (6) with respect to $\phi(\mathbf{x})$. Those are calculated in the following section.

\subsection{Functional derivatives of $\boldsymbol{\Psi}$}

By using the chain rule for the functional differentiation and introducing the notations

$$
\boldsymbol{\Phi}(\mathbf{x}):=\frac{\delta \mathbf{\Psi}}{\delta \Psi_{\Sigma}^{T}(\mathbf{x})}
$$

and

$$
\partial_{\phi} \Psi_{\Sigma}(\mathbf{x}):=\left.(\partial \Psi / \partial \phi)\right|_{\Sigma}(\mathbf{x}), \quad \partial_{\phi \phi} \Psi_{\Sigma}(\mathbf{x}):=\left.\left(\partial^{2} \Psi / \partial \phi^{2}\right)\right|_{\Sigma}(\mathbf{x}),
$$


we obtain

$$
\begin{aligned}
\frac{\delta \boldsymbol{\Psi}}{\delta \phi(\mathbf{x})}= & \operatorname{Tr}\left\{\boldsymbol{\Phi}(\mathbf{x}) \partial_{\phi} \Psi_{\Sigma}(\mathbf{x})\right\}+\frac{\bar{\delta} \boldsymbol{\Psi}}{\bar{\delta} \phi(\mathbf{x})} \\
\frac{\delta^{2} \boldsymbol{\Psi}}{\delta \phi(\mathbf{x})^{2}}= & \operatorname{Tr}\left\{\delta(\mathbf{0}) \boldsymbol{\Phi}(\mathbf{x}) \partial_{\phi \phi} \Psi_{\Sigma}(\mathbf{x})+2 \frac{\bar{\delta} \boldsymbol{\Phi}(\mathbf{x})}{\delta \phi(\mathbf{x})} \partial_{\phi} \Psi_{\Sigma}(\mathbf{x})\right\} \\
& +\operatorname{Tr} \operatorname{Tr}\left\{\frac{\delta^{2} \boldsymbol{\Psi}}{\delta \Psi_{\Sigma}^{T}(\mathbf{x}) \otimes \delta \Psi_{\Sigma}^{T}(\mathbf{x})} \partial_{\phi} \Psi_{\Sigma}(\mathbf{x}) \otimes \partial_{\phi} \Psi_{\Sigma}(\mathbf{x})\right\}+\frac{\bar{\delta}^{2} \boldsymbol{\Psi}}{\bar{\delta} \phi(\mathbf{x})^{2}}
\end{aligned}
$$

\subsubsection{The potential term $\mathrm{V}$}

Our starting observation will be that the term $V$ in (14) has to reproduce the last term in the functional derivative Schrödinger equation (1). This means that there exists a mapping $\mapsto$ such that

$$
\left.\int \mathrm{d} \mathbf{x} \operatorname{Tr}\left\{\boldsymbol{\Phi}(\mathbf{x}) \frac{1}{\varkappa} \gamma_{0} V(\phi(\mathbf{x})) \Psi_{\Sigma}(\mathbf{x})\right)\right\} \mapsto \int \mathrm{d} \mathbf{x} \sqrt{g} V(\phi(\mathbf{x})) \mathbf{\Psi} .
$$

The existence of the map in (19) implies that the following relation should be fulfilled at any spatial point $\mathbf{x}$ :

$$
\operatorname{Tr}\left\{\boldsymbol{\Phi}(\mathbf{x}) \frac{1}{\varkappa} \gamma_{0} \Psi_{\Sigma}(\mathbf{x})\right\} \mapsto \sqrt{g} \mathbf{\Psi} .
$$

Then the functional differentiation of both sides of (20) with respect to $\Psi_{\Sigma}^{T}(\mathbf{x})$ yields

$$
\operatorname{Tr}\left\{\frac{\delta^{2} \boldsymbol{\Psi}}{\delta \Psi_{\Sigma}^{T}(\mathbf{x}) \otimes \delta \Psi_{\Sigma}^{T}(\mathbf{x})} \frac{1}{\varkappa} \gamma_{0} \Psi_{\Sigma}(\mathbf{x})\right\}+\boldsymbol{\Phi}(\mathbf{x}) \frac{1}{\varkappa} \gamma_{0} \delta(\mathbf{0}) \mapsto \sqrt{g} \boldsymbol{\Phi}(\mathbf{x}),
$$

where again, $\delta(\mathbf{0})=\delta \Psi_{\Sigma}(\mathbf{x}) / \delta \Psi_{\Sigma}^{T}(\mathbf{x})$. This type of relation is possible if

$$
\frac{\delta^{2} \mathbf{\Psi}}{\delta \Psi_{\Sigma}^{T}(\mathbf{x}) \otimes \delta \Psi_{\Sigma}^{T}(\mathbf{x})}=0
$$

and

$$
\frac{1}{\varkappa} \gamma_{0}(x) \delta(\mathbf{0})-\sqrt{g}(x) \mapsto 0 .
$$

The latter relation can be understood as the condition

$$
\gamma^{0} \sqrt{g} \varkappa \mapsto \delta(\mathbf{0})
$$

By taking into account that $\sqrt{g}=\sqrt{g_{00} h}$, where $h:=\left|\operatorname{det}\left(g_{i j}\right)\right|$, and $\gamma^{0} \sqrt{g_{00}}=\gamma_{0}$ is the time-like tangent Minkowski space Dirac matrix, eq. (24) can be rewritten as

$$
\underline{\gamma}_{0} \varkappa \mapsto \delta(\mathbf{0}) / \sqrt{h}=\delta^{\text {inv }}(\mathbf{0}),
$$


where $\delta^{\operatorname{inv}}(\mathbf{x})$ is the invariant $(n-1)$-dimensional delta function defined by the property $\int d \mathbf{x} \sqrt{h}(\mathbf{x}) \delta^{i n v}(\mathbf{x})=1$. This formula generalizes to curved space-times the limiting map $\gamma_{0} \varkappa \mapsto \delta(\mathbf{0})$ already found in flat space-time $[29,30]$, with the $(n-1)$-dimensional delta function replaced by the invariant one.

\subsubsection{The second variational derivative term}

Our next observation is that the term $I V$ in (14) is able to reproduce the first term in the right-hand side of (18) in the limiting case (24)

$$
I V: \quad-\frac{\varkappa}{2} \gamma_{0} \partial_{\phi \phi} \Psi_{\Sigma} \mapsto-\frac{1}{\sqrt{g}} g_{00} \delta(\mathbf{0}) \partial_{\phi \phi} \Psi_{\Sigma}
$$

A comparison with (18) shows that the term $I V$ in (14) leads to the following expression in functional derivatives of $\Psi$ :

$$
I V: \operatorname{Tr}\left\{\frac{1}{2} \boldsymbol{\Phi}(\mathbf{x}) \varkappa \gamma_{0} \partial_{\phi \phi} \Psi_{\Sigma}(\mathbf{x})\right\} \mapsto \frac{1}{2} \frac{g_{00}}{\sqrt{g}}\left(\frac{\delta^{2} \mathbf{\Psi}}{\delta \phi(\mathbf{x})^{2}}-2 \operatorname{Tr}\left\{\frac{\bar{\delta} \mathbf{\Phi}(\mathbf{x})}{\bar{\delta} \phi(\mathbf{x})} \partial_{\phi} \Psi_{\Sigma}(\mathbf{x})\right\}-\frac{\bar{\delta}^{2} \mathbf{\Psi}}{\bar{\delta} \phi(\mathbf{x})^{2}}\right) .
$$

The first term in the right-hand side of (27) correctly reproduces the first term in the functional derivative Schrödinger equation (1). However, the second and the third term need further investigation.

3.4.3. The non-ultralocality term and the wave functional $\Psi$ in terms of precanonical $\Psi_{\Sigma}$

Since the right hand side of (14) is expected to lead to a functional derivative operator acting on the wave functional $\Psi$, as in the right hand side of the functional Schrödinger equation (1), the second term in (27) with $\partial_{\phi} \Psi_{\Sigma}$ has to be cancelled by the term II in (14) which also contains $\partial_{\phi} \Psi_{\Sigma}$. Therefore, it is required that

$$
I I+2 \text { nd term of }(27): \quad \mathrm{i} \boldsymbol{\Phi}(\mathbf{x}) \gamma_{0} \gamma^{i} \partial_{i} \phi(\mathbf{x})+\frac{g_{00}}{\sqrt{g}} \frac{\bar{\delta} \boldsymbol{\Phi}(\mathbf{x})}{\delta \phi(\mathbf{x})} \mapsto 0,
$$

where the sign $\mapsto$ stresses the fact that it is sufficient that the left hand side vanishes under the condition (24) rather than as an equality. In fact, by functionally differentiating both sides of (28) with respect to $\phi\left(\mathbf{x}^{\prime}\right)$ we can see that (28) with $\mapsto$ replaced by the equality is not an integrable equation in functional derivatives. Nevertheless, by bearing in mind that (28) has to be valid only under the condition (24), the solution for $\boldsymbol{\Phi}(\mathbf{x})$ can be written in the form

$$
\boldsymbol{\Phi}(\mathbf{x})=\boldsymbol{\Xi}\left(\left[\Psi_{\Sigma}\right] ; \check{\mathbf{x}}\right) e^{-\mathrm{i} \phi(\mathbf{x}) \gamma^{i} \partial_{i} \phi(\mathbf{x}) / \varkappa},
$$

where the "integration constant" $\Xi\left(\left[\Psi_{\Sigma}\right] ; \check{\mathbf{x}}\right)$ is a functional of $\Psi_{\Sigma}\left(\mathbf{x}^{\prime}\right)$ on the punctured space with the removed point $\mathbf{x}$ such that that $\mathbf{x}^{\prime} \neq \mathbf{x}$. By construction, this functional satisfies the identity

$$
\frac{\bar{\delta} \Xi\left(\left[\Psi_{\Sigma}\right] ; \check{\mathbf{x}}\right)}{\bar{\delta} \phi(\mathbf{x})} \equiv 0
$$

Indeed, by differentiating (29) with respect to $\phi(\mathbf{x})$, replacing $\varkappa$ according to the limiting map (24), and taking into account that $\gamma_{0}(\mathbf{x}) \gamma_{0}(\mathbf{x})=: g_{00}(\mathbf{x})$ and $\partial_{i} \delta(\mathbf{0})=0$ (that restricts the admissible class of regularizatons of delta-function $\delta(\mathbf{x})$ ) we conclude that (29) solves (28) under the condition (24). Note also that (29) by construction fulfills

$$
\frac{\delta \Phi(\mathbf{x})}{\delta \Psi_{\Sigma}^{T}(\mathbf{x})}=\frac{\delta^{2} \Psi}{\delta \Psi_{\Sigma}^{T}(\mathbf{x}) \otimes \delta \Psi_{\Sigma}^{T}(\mathbf{x})} \equiv 0
$$


which is consistent with (22). Thus the required cancellation of the terms with $\partial_{\phi} \Psi_{\Sigma}(\mathbf{x})$ (under the

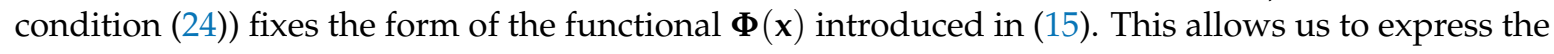
wave functional $\Psi$ in the form

$$
\boldsymbol{\Psi} \sim \operatorname{Tr}\left\{\boldsymbol{\Xi}\left(\left[\Psi_{\Sigma}\right] ; \check{\mathbf{x}}\right) e^{-\mathbf{i} \phi(\mathbf{x}) \gamma^{i} \partial_{i} \phi(\mathbf{x}) / \varkappa} \frac{\gamma_{0}}{\sqrt{g} \varkappa} \Psi_{\Sigma}(\mathbf{x})\right\}_{\left.\right|_{\varkappa \longmapsto \gamma_{0} \delta(\mathbf{0}) / \sqrt{g}}},
$$

valid at any point $\mathbf{x}$. Here the equality up to a normalization factor which will depend on $\varkappa$ and $\sqrt{g}$ is denoted as $\sim$. The notation $\left.\{\ldots\}\right|_{\left.\right|_{\varkappa} \longmapsto \gamma_{0} \delta(\mathbf{0}) / \sqrt{g}}$ indicates that every appearance of $\varkappa$ in the expression inside braces is replaced by $\gamma_{0} \delta(\mathbf{0}) / \sqrt{g}$ as prescribed by the limiting map (24).

Using (31) we can now evaluate the last term in (27) in the limit (24):

$$
\text { 3-rd term of (27): } \quad \frac{1}{2} \frac{g_{00}}{\sqrt{g}} \frac{\bar{\delta}^{2} \mathbf{\Psi}}{\bar{\delta} \phi(\mathbf{x})^{2}} \mapsto-\frac{1}{2} \sqrt{g} g^{i j} \partial_{i} \phi(\mathbf{x}) \partial_{j} \phi(\mathbf{x}) \Psi .
$$

The right hand side of (32) correctly reproduces the second term in the functional derivative Schrödinger equation (1), thus correctly accounting for the inherent non-ultralocality of quantum relativistic scalar field theory (cf. [42]) in curved space-time.

Thus, all terms in the functional derivative Schrödinger equation (1) are now derived from pSE restricted to $\Sigma$, eq. (8). However, there are still unaccounted for terms I, III $a$ and IIIb in (14)

$$
I+I I I a+I I I b: \quad-\mathrm{i} \int \mathrm{d} \mathbf{x} \operatorname{Tr}\left\{\boldsymbol{\Phi}(\mathbf{x}) \gamma_{0} \gamma^{i} \nabla_{i}^{t o t} \Psi_{\Sigma}+\boldsymbol{\Phi}(\mathbf{x})\left[\omega_{0}, \Psi_{\Sigma}\right]\right\} .
$$

In flat space-time [29-31], those terms are reduced to the term $I$ with with the total derivative $d \Psi_{\Sigma}(\mathbf{x}) / d x^{i}$ which does not contribute to the equation for the functional $\Psi$ if $\Psi_{\Sigma}(\mathbf{x})$ vanishes at the spatial infinity. Let us see if or how this property extends to curved space-times.

\subsubsection{The vanishing contribution from the terms I and IIIa}

At first we consider the first term in (33). Using the covariant Stokes theorem we obtain

$$
\begin{aligned}
I+I I I a: \quad-\mathrm{i} \int \mathrm{d} \mathbf{x} \operatorname{Tr}\left\{\boldsymbol{\Phi}(\mathbf{x}) \gamma_{0} \gamma^{i} \nabla_{i}^{\text {tot }} \Psi_{\Sigma}\right\}=-\mathrm{i} \int \mathrm{d} \mathbf{x} \sqrt{h}\left(\operatorname{Tr}\left\{\frac{1}{\sqrt{h}} \boldsymbol{\Phi}(\mathbf{x}) \gamma_{0} \gamma^{i} \nabla_{i}^{\text {tot }} \Psi_{\Sigma}\right\}\right) \\
=-\mathrm{i} \int \mathrm{d} \mathbf{x} \sqrt{h} \operatorname{Tr}\left\{\nabla_{i}^{\text {tot }}\left(\frac{1}{\sqrt{h}} \boldsymbol{\Phi}(\mathbf{x}) \gamma_{0} \gamma^{i} \Psi_{\Sigma}\right)\right\}+\mathrm{i} \int \mathrm{d} \mathbf{x}\left(\sqrt{h} \operatorname{Tr}\left\{\nabla_{i}^{\text {tot }}\left(\frac{1}{\sqrt{h}} \boldsymbol{\Phi}(\mathbf{x}) \gamma_{0} \gamma^{i}\right) \Psi_{\Sigma}\right\}\right) \\
=-\mathrm{i} \oint_{\partial \Sigma} \mathrm{d} \mathbf{x}_{i} \operatorname{Tr}\left\{\boldsymbol{\Phi} \gamma_{0} \gamma^{i} \Psi_{\Sigma}\right\}+\mathrm{i} \int \mathrm{d} \mathbf{x} \operatorname{Tr}\left\{\boldsymbol{\Phi}\left(\nabla_{i}^{\text {tot }}\left(\gamma_{0} \gamma^{i}\right)\right) \Psi_{\Sigma}\right\} \\
+\mathrm{i} \int \mathrm{d} \mathbf{x}\left(\frac{-\nabla_{i} \sqrt{h}}{\sqrt{h}} \operatorname{Tr}\left\{\boldsymbol{\Phi} \gamma_{0} \gamma^{i} \Psi_{\Sigma}\right\}+\operatorname{Tr}\left\{\left(\nabla_{i}^{\text {tot }} \boldsymbol{\Phi}(\mathbf{x})\right) \gamma_{0} \gamma^{i} \Psi_{\Sigma}\right\}\right)
\end{aligned}
$$

where $\mathrm{d} \mathbf{x}_{i}=\left.d^{n-2} \mathbf{x}\right|_{\partial \Sigma} n_{i}(\mathbf{x})$ is the measure of $(n-2)$-dimensional integration over the boundary $\partial \Sigma$ with the normal vector $n_{i}(\mathbf{x})$ tangent to $\Sigma$. In the right hand side of (34),

(i) the first boundary term is the result of the covariant Stokes theorem and it vanishes if $\Psi_{\Sigma}$ vanishes on the boundary $\partial \Sigma$;

(ii) the following three terms follow from the Leibniz rule for the total covariant derivative $\nabla_{i}^{\text {tot }}$ with respect to the Clifford products of tensor Clifford-algebra-valued functions;

(iii) in the second term, $\nabla_{i}^{\text {tot }}\left(\gamma_{0} \gamma^{i}\right)=0$ due to the covariant constancy of Dirac matrices (12);

(iv) in the third term, the metric compatibility yields $\nabla_{i} \sqrt{h}=0$;

(v) in the fourth term, the explicit formula for $\boldsymbol{\Phi}(\mathbf{x})$ in (29) yields

$$
\nabla_{i}^{t o t} \boldsymbol{\Phi}(\mathbf{x})=\frac{-\mathrm{i}}{\varkappa} \boldsymbol{\Phi}(\mathbf{x})\left(\partial_{i} \phi \gamma^{l} \partial_{l} \phi+\phi \gamma^{l} \partial_{i l} \phi+\phi\left(\nabla_{i}^{t o t} \gamma^{l}\right) \partial_{l} \phi\right) .
$$


By noticing that the last term in (35) vanishes due to (12) and substituting (35) into the last term in (34), using the covariant Stokes theorem and the assumption that the field configurations $\phi(\mathbf{x})$ vanish at the spatial infinity, we obtain

$$
\begin{aligned}
\int \mathrm{d} \mathbf{x} \operatorname{Tr}\left\{\boldsymbol{\Phi}(\mathbf{x}) \frac{1}{\varkappa} \gamma_{0} \Psi_{\Sigma}(\mathbf{x})\left(g^{i l} \partial_{i} \phi \partial_{l} \phi+\phi g^{i l} \partial_{i l} \phi\right)\right\} & =\mathbf{\Psi} \int \mathrm{d} \mathbf{x} \sqrt{g}\left(g^{i l} \partial_{i} \phi \partial_{l} \phi+\phi g^{i l} \partial_{i l} \phi\right) \\
& =-\mathbf{\Psi} \int \mathrm{d} \mathbf{x} \sqrt{h} \nabla_{i}\left(\sqrt{g_{00}} g^{i l}\right) \frac{1}{2} \partial_{l} \phi^{2}=0,
\end{aligned}
$$

where we use in the first equality the fact that $\operatorname{Tr}\left\{\boldsymbol{\Phi}(\mathbf{x}) \frac{1}{\varkappa \sqrt{8}} \gamma_{0} \Psi_{\Sigma}(\mathbf{x})\right\}=\mathbf{\Psi}$ (c.f. (20)) and the covariant Stokes theorem in the second equality. The result is that the right-hand side of (36) vanishes because of the metricity of space-time: $\nabla_{\alpha} g^{\mu v}=0$.

Therefore, it is demonstrated that in the limiting case (24) all four terms in the right-hand side of (34) vanish, so that the terms I and IIIa in (14) do not contribute to the equation for the functional $\Psi$.

By combining the above considerations we obtain from (14) the following equation for the functional $\Psi$ :

$$
\mathrm{i} \hbar \partial_{t} \mathbf{\Psi}=\int d \mathbf{x} \sqrt{g}\left(\frac{\hbar^{2}}{2} \frac{g_{00}}{g} \frac{\delta^{2}}{\delta \phi(\mathbf{x})^{2}}-\frac{1}{2} g^{i j} \partial_{i} \phi(\mathbf{x}) \partial_{j} \phi(\mathbf{x})+V(\phi)\right) \mathbf{\Psi}-\frac{\mathrm{i}}{4} \operatorname{Tr}\left\{\boldsymbol{\Phi}(\mathbf{x})\left[\omega_{0}, \Psi_{\Sigma}\right]\right\}
$$

We see that the first three terms in the right hand side reproduce the canonical Hamiltonian operator in the functional derivative Schrödinger equation (1). However, the last term, which does not vanish in arbitrary non-static space-times where $\omega_{0} \neq 0$, still can not be expressed in terms of $\Psi$ alone. For this reason, we will treat static space-times with $\omega_{0}=0$ and non-static ones with $\omega_{0} \neq 0$ separately.

\section{Static space-times with $\omega_{0}=0$}

In static space-times, when $\omega_{0}=0$, equation (37) coincides with the canonical functional derivative Schrödinger equation (1). Thus the latter is derived from the precanonical Schrödinger equation as the limiting case corresponding to (24). In this case, we can also specify the functional $\Xi\left(\left[\Psi_{\Sigma}(\mathbf{x})\right], \check{\mathbf{x}}\right)$ in (31) by combining the observations presented above together and noticing that the relation (31) is valid at any given point $\mathbf{x}$. This is possible only if the functional $\mathbf{\Psi}$ is the continual product of identical terms at all points $\mathbf{x}$, namely,

$$
\left.\mathbf{\Psi} \sim \operatorname{Tr}\left\{\prod_{\mathbf{x}} e^{-i \phi(\mathbf{x}) \gamma^{i} \partial_{i} \phi(\mathbf{x}) / \varkappa} \underline{\gamma}_{0} \Psi_{\Sigma}(\phi(\mathbf{x}), \mathbf{x}, t)\right\}\right|_{\varkappa \mapsto \gamma_{0} \delta(\mathbf{0}) / \sqrt{g}},
$$

where $\sim$ means an equality up to a normalization factor which includes $\varkappa$ and $\sqrt{h}$.

The formal continual product expression in (38) can be understood as the multidimensional product integral $[37,38]$

$$
\left.\mathbf{\Psi} \sim \operatorname{Tr}\left\{\int_{\mathbf{x}} e^{-i \phi(\mathbf{x}) \gamma^{i}(\mathbf{x}) \partial_{i} \phi(\mathbf{x}) / \varkappa} \underline{\gamma}_{0} \Psi_{\Sigma}(\phi(\mathbf{x}), \mathbf{x}, t)\right\}\right|_{\frac{1}{\varkappa} \underline{\gamma}_{0} \mapsto \sqrt{h} \mathbf{d} \mathbf{x}^{\prime}}
$$

where the notation of the product integral of matrix-valued functions $F(\mathbf{x})$ as proposed by R. Gill [39] (and implemented in the $\mathrm{LT}_{\mathrm{E}} \mathrm{X}$ package prodint) is used

$$
\pi_{\mathbf{x}} e^{F(\mathbf{x}) \mathrm{d} \mathbf{x}}=\pi_{\mathbf{x}}(1+F(\mathbf{x}) \mathrm{d} \mathbf{x}) .
$$

The expression in (39) generalizes a similar result obtained in flat space-time earlier [30]. The only difference is that in curved space-time the spatial integration measure $\mathrm{dx}$ is replaced by the invariant one $\sqrt{h} \mathrm{~d} \mathbf{x}$ and the Dirac matrices are $\mathbf{x}$-dependent. 
In $(1+1)$-dimensional space-time, the product integral above is given by the well known path-ordered exponential, or the Peano-Baker series (also known as the Dyson series in the context of perturbative QFT and the path-ordered phase related to the Wilson loop in gauge theory), cf. eq. (53) below. A multidimensional generalization is briefly discussed in the books [37,38] and probably needs further refinement. However, in our case, instead of defining the product integral of arbitrary non-commutative matrices, we need only the trace of the product integral of Clifford-algebra valued functions. This significantly simplifies the task of defining the expression (39) mathematically. For example, in the one-dimensional case, the taking of the trace of each of the terms in the series expansion of the ordered exponential in (53)) implies that the matrices under the integrals in the series expansion of the trace of product integral are multiplied in the cycling permuted way, which can be generalized to the multidimensional case, rather than a time-ordered one, which implies a one-parameter ordering whose multidimensional generalization is problematic. Then, if the corresponding limit exists,

$$
\operatorname{Tr} \int_{\mathbf{x} \in V} e^{F(\mathbf{x}) \mathrm{d} \mathbf{x}}:=\lim _{N \rightarrow \infty} \frac{1}{N !} \operatorname{Tr} \sum_{P(N)} e^{F\left(\mathbf{x}_{1}\right) \Delta \mathbf{x}_{1}} e^{F\left(\mathbf{x}_{2}\right) \Delta \mathbf{x}_{2}} \ldots e^{F\left(\mathbf{x}_{N}\right) \Delta \mathbf{x}_{N}},
$$

By substituting $\Psi_{\Sigma}$ in the form

$$
\Psi_{\Sigma}=e^{+\frac{\mathrm{i}}{\varkappa} \phi(\mathbf{x}) \gamma^{i} \partial_{i} \phi(\mathbf{x})} \Phi_{\Sigma}
$$

we obtain

$$
\mathrm{i} \partial_{t} \Psi_{\Sigma}=e^{+\frac{\mathrm{i}}{\varkappa} \phi(\mathbf{x}) \gamma^{i} \partial_{i} \phi(\mathbf{x})} \mathrm{i} \partial_{t} \Phi_{\Sigma}
$$

in the left hand side of (42) and

$$
\gamma_{0} e^{+\frac{i}{\varkappa} \phi(\mathbf{x}) \gamma^{i} \partial_{i} \phi(\mathbf{x})}\left(-\frac{\varkappa}{2} \partial_{\phi \phi}-\frac{1}{2 \varkappa} g^{i j}(\mathbf{x}) \partial_{i} \phi(\mathbf{x}) \partial_{j} \phi(\mathbf{x})+\frac{1}{\varkappa} V(\phi)\right) \Phi_{\Sigma}
$$

in the right hand side. Hence, $\Phi_{\Sigma}$ obeys

$$
\mathrm{i} \partial_{t} \Phi_{\Sigma}=\widetilde{\gamma}_{0}(\mathbf{x})\left(-\frac{\varkappa}{2} \partial_{\phi \phi}-\frac{1}{2 \varkappa} g^{i j}(\mathbf{x}) \partial_{i} \phi(\mathbf{x}) \partial_{j} \phi(\mathbf{x})+\frac{1}{\varkappa} V(\phi)\right) \Phi_{\Sigma},
$$

where

$$
\widetilde{\gamma}_{0}(\mathbf{x}):=e^{-\frac{\mathrm{i}}{\varkappa} \phi(\mathbf{x}) \gamma^{i} \partial_{i} \phi(\mathbf{x})} \gamma_{0}(\mathbf{x}) e^{+\frac{\mathrm{i}}{\varkappa} \phi(\mathbf{x}) \gamma^{i} \partial_{i} \phi(\mathbf{x})} .
$$

Obviously, $\widetilde{\gamma}_{0}(\mathbf{x}) \widetilde{\gamma}_{0}(\mathbf{x})=\gamma_{0}(\mathbf{x}) \gamma_{0}(\mathbf{x})=g_{00}(\mathbf{x})$, hence the transformation in (47) is a Clifford algebra isomorphism.

From (46) one can conclude that $\Phi_{\Sigma}$ can be written in the form

$$
\Phi_{\Sigma}=\left(1+\underline{\gamma}^{0}\right) \Phi_{\Sigma}^{\times},
$$


where $\Phi_{\Sigma}^{\times}$is a scalar function such that

$$
\mathrm{i} \partial_{t} \Phi_{\Sigma}^{\times}=\sqrt{g_{00}}\left(-\frac{\varkappa}{2} \partial_{\phi \phi}-\frac{1}{2 \varkappa} g^{i j}(\mathbf{x}) \partial_{i} \phi(\mathbf{x}) \partial_{j} \phi(\mathbf{x})+\frac{1}{\varkappa} V(\phi)\right) \Phi_{\Sigma}^{\times} .
$$

In terms of scalar function $\Phi_{\Sigma}^{\times}$eq. (39) takes the form

$$
\left.\mathbf{\Psi} \sim \operatorname{Tr}\left\{\int_{\mathbf{x}}\left(1+\underline{\gamma}_{0}\right) \Phi_{\Sigma}^{\times}(\phi(\mathbf{x}), \mathbf{x}, t)\right\}\right|_{\frac{1}{\varkappa} \underline{\gamma}_{0} \mapsto \sqrt{h} \mathbf{d} \mathbf{x}} \sim \int_{\mathbf{x}} \Phi_{\Sigma}^{\times}\left(\phi(\mathbf{x}), \mathbf{x},\left.t\right|_{\frac{1}{\varkappa} \mapsto \sqrt{h} \mathbf{d} \mathbf{x}},\right.
$$

where we use the projector property of the matrix $\left(1+\underline{\gamma}_{0}\right)$. Obviously, the multidimensional product integral of the scalar function $\Phi_{\Sigma}^{\times}$is defined without any complications related to the definition of the product integral of non-commutative matrix functions.

\section{Non-static space-times with $\omega_{0} \neq 0$}

In non-static space-times, when $\omega_{0} \neq 0$, the last term in (37) does not allow us to obtain a close equation for the functional $\Psi$. In order to find a way out, let us write the effective equation similar to (42) which governs the time evolution of $\Psi_{\Sigma}$, with the term $I$ and the spatial part of the term IIIa in (14), which are proven in (34) to have no contribution to $\partial_{t} \Psi$, removed:

$$
\left.\mathrm{i} \partial_{t} \Psi_{\Sigma}=\gamma_{0}\left(-\frac{\varkappa}{2} \partial_{\phi \phi}+\mathrm{i} \gamma^{i} \partial_{i} \phi(\mathbf{x}) \partial_{\phi}+\frac{1}{\varkappa} V(\phi)\right)\right) \Psi_{\Sigma}-\mathrm{i}\left[\omega_{0}, \Psi_{\Sigma}\right]=: \hat{H}_{0}-\mathrm{i}\left[\omega_{0}, \Psi_{\Sigma}\right]
$$

We first note that by transforming $\Psi_{\Sigma}$ as follows:

$$
\Psi_{\Sigma}:=U \Psi_{\Sigma}^{\prime} U^{-1}
$$

where

$$
\begin{aligned}
U(\mathbf{x}, t) & =\mathcal{T} e^{-\int_{0}^{t} d s \omega_{0}(\mathbf{x}, s)}=\prod_{t=0}^{t}\left(1-\omega_{0}(\mathbf{x}, s)\right) d s \\
& :=1-\int_{0}^{t} d t_{1} \omega_{0}\left(\mathbf{x}, t_{1}\right)+\int_{0}^{t} d t_{1} \int_{0}^{t_{1}} d t_{2} \omega_{0}\left(\mathbf{x}, t_{1}\right) \omega_{0}\left(\mathbf{x}, t_{2}\right) \\
& -\int_{0}^{t} d t_{1} \int_{0}^{t_{1}} d t_{2} \int_{0}^{t_{2}} d t_{3} \omega_{0}\left(\mathbf{x}, t_{1}\right) \omega_{0}\left(\mathbf{x}, t_{2}\right) \omega_{0}\left(\mathbf{x}, t_{3}\right)+\ldots
\end{aligned}
$$

is the tranformation determined by the time-ordered exponential, we obtain

$$
\mathrm{i} \partial_{t} \Psi=-\mathrm{i}\left[\omega_{0}, \Psi\right]+U \mathrm{i} \partial_{t} \Psi_{\Sigma}^{\prime} U^{-1} .
$$

Then

$$
\mathrm{i} \partial_{t} \Psi_{\Sigma}^{\prime}=U^{-1} \hat{H}_{0} \Psi_{\Sigma} U=\hat{H}_{0}^{\prime} \Psi^{\prime}
$$

where

$$
\Psi^{\prime}:=U^{-1} \Psi_{\Sigma} U, \quad \hat{H}_{0}^{\prime}:=U^{-1} \hat{H}_{0} U .
$$

As the transformation $U$ affects only the terms with $\gamma^{\mu}$-s,

$$
\left.\hat{H}_{0}{ }^{\prime}=\gamma_{0}^{\prime}\left(-\frac{\varkappa}{2} \partial_{\phi \phi}+\mathrm{i} \gamma^{\prime i} \partial_{i} \phi(\mathbf{x}) \partial_{\phi}+\frac{1}{\varkappa} V(\phi)\right)\right),
$$

where

$$
\gamma^{\prime \mu}(\mathbf{x}, t):=U^{-1}(\mathbf{x}, t) \gamma^{\mu}(x) U(\mathbf{x}, t) .
$$


It is easy to check that

$$
\gamma^{\prime \mu} \gamma^{\prime \nu}+\gamma^{\prime \nu} \gamma^{\prime \mu}=2 U^{-1} g^{\mu \nu} U=2 g^{\mu \nu}
$$

Hence the $U$-transformation is just a local automorphism of the Clifford algebra of space-time.

Using (55) one can write

$$
\begin{aligned}
\mathrm{i} \partial_{t} \Psi & =\operatorname{Tr} \int d \mathbf{x} \frac{\delta \boldsymbol{\Psi}}{\delta \Psi_{\Sigma}^{\prime T}(\mathbf{x})} \mathrm{i} \partial_{t} \Psi_{\Sigma}^{\prime} \\
& =\operatorname{Tr} \int d \mathbf{x} \frac{\delta \Psi}{\delta \Psi^{\prime \prime}(\mathbf{x})} \hat{H}_{0}^{\prime} \Psi_{\Sigma}^{\prime} .
\end{aligned}
$$

By comparing it with (7) and (8) we conclude that the results in static space-times with $\omega_{0}=0$ are generalized to non-static space-times with $\omega_{0} \neq 0$ using the dictionary:

$$
\begin{aligned}
\gamma^{\mu} & \rightarrow \gamma^{\prime \mu}=U^{-1} \gamma^{\mu} U, \\
\Psi_{\Sigma} & \rightarrow \Psi_{\Sigma}^{\prime}=U^{-1} \Psi_{\Sigma} U, \\
\hat{H}_{0 \Sigma} & \rightarrow \hat{H}_{0 \Sigma}^{\prime}=U^{-1} H_{0 \Sigma} U
\end{aligned}
$$

with $U$ given by the path-ordered exponential in (53). Then, the wave functional (39) rewritten in terms of the primed objects:

$$
\left.\mathbf{\Psi} \sim \operatorname{Tr}\left\{\int_{\mathbf{x}} e^{-i \phi(\mathbf{x}) \gamma^{\prime i}(\mathbf{x}, t) \partial_{i} \phi(\mathbf{x}) / \varkappa} \underline{\gamma}_{0} \Psi_{\Sigma}^{\prime}(\phi(\mathbf{x}), \mathbf{x}, t)\right\}\right|_{\underline{\gamma}_{0} 0 \frac{1}{\varkappa} \mapsto \sqrt{h} \mathrm{~d} \mathbf{x}}
$$

represents, up to a normalization factor, the Schrödinger wave functional in terms of precanonical wave functions in an arbitrary curved space-time and it satisfies (37) without the last term, i.e. the functional derivative Schrödinger equation (1). Using the same steps as in the static case, this complicated expression can be transformed to the product integral of the scalar function $\Phi_{\Sigma}^{\times}$,

$$
\left.\mathbf{\Psi} \sim \pi_{\mathbf{x}} \Phi_{\Sigma}^{\times}(\phi(\mathbf{x}), \mathbf{x}, t)\right|_{\frac{1}{\tilde{h}} \mapsto \sqrt{h} \mathrm{~d} \mathbf{x}^{\prime}}
$$

the only difference being the metric tensor components in eq. (49) defining $\Phi_{\Sigma}^{\times}$can now be also time-dependent.

In summary, we have demonstrated that in curved space-times the canonical functional derivative Schrödinger equation (1) and the explicit product integral formula (39) relating the Schrödinger wave functional with the Clifford-valued precanonical wave function can be derived from the precanonical Schrödinger equation (2) in the singular limiting case when $\gamma_{0} \varkappa$ is replaced by $\delta(\mathbf{0}) / \sqrt{h}$, a regularized invariant delta-function at coinciding spatial points. A natural interpretation of the latter is that it represents the UV cutoff of the total volume of the momentum space which one has to introduce in order to make sense of the second variational derivative at coincinding points in (1). As in the previously considered case of quantum fields in flat space-time [29-31], the standard unregularized formulation of QFT in curved space-time in functional Schrödinger representation thus emerges from the precanonical description as a singular limiting case.

\section{Conclusion}

We explored a connection between the description of an interacting quantum scalar field in curved space-time derived from precanonical quantization and the standard description in the functional Schrödinger picture resulting from the canonical quantization.

We have demonstrated that the functional derivative Schrödinger equation (1) can be derived from the partial derivative precanonical Schrödinger equation (2) in the limiting case (24). Namely, the restriction of the precanonical Schrödinger equation to the subspace $\Sigma$ representing a field configuration 
at time $t$, eq. (8), governs the time evolution of the wave functional according to (7) and (14). Then, in the limiting case (24),

(i) the potential term $V$ in (14) reproduces the potential term in (1);

(ii) the term $I V$ in (14) reproduces the second functional derivative term in (1) up to some additional terms which have no obvious counterpart in (1);

(iii) by noticing that one of those additional terms can be cancelled by the term II in (14) we obtain an expression of the Schrödinger wave functional as a trace of the continuous product of the precanonical wave functions (38) which we suggested to interpret as a multidimensional analogue of the product integral, eq. (39);

(iv) using the expression of the wave functional in terms of pre3canonical wave functions in the other additional term mentioned in (ii) we reproduce the second term in the right-hand side of (1);

(v) this explicit expression also allows us to show that for the fields $\phi(\mathbf{x})$ and $\Psi_{\Sigma}(\phi(\mathbf{x}), \mathbf{x}, t)$ vanishing at the spatial infinity the remaining terms $I$ and IIIa do not contribute to the functional Schrödinger equation (1);

(vi) in static space-times when $\omega_{0}=0$ the remaining term $I I I b$ vanishes and the functional Schrödinger equation (1) emerges from the precanonical Schrödinger equation (2) and the Schrödinger wave functional is expressed in terms of precanonical wave functions;

(vii) in non-static space-times with $\omega_{0} \neq 0$, we argue that the transformation (53) absorbs the contribution of the term IIIb in (14) thus allowing us to obtain the functional Schrödinger equation (1) from the precanonical Schrödinger equation (2) and to express the Schrödinger wave functional in terms of transformed precanonical wave functions, eq. (66);

(viii) both in static and non-static space-times the Schrödinger wave functional can be represented as the product integral of a scalar wave function derived from the precanonical wave function by a series of transformations and satisfying equation (49).

These results generalize to arbitrary curved space-times (whose metric is represented in Gaussian coordinates with $g_{0 i}=0$ ) the statement from [29-31] that the standard functional Schrödinger representation of QFT is a certain (symbolic) limiting case of the theory of quantum fields obtained by precanonical quantization.

The symbolic or singular nature of the limiting transition from precanonical quantization to the standard formulation of QFT in functional Schrödinger representation is related to the fact that the latter, due to the presence of the second functional derivative at coinciding points, is not a well-defined theory unless a regularization is introduced. The regularization typically introduces a UV cutoff scale as an additional element of the theory removed by a subsequent renormalization. In precanonical quantization, the ultraviolet scale $\varkappa$ appears as an inherent element quantization, which, unlike other theories introducing an ultraviolet fundamental length or cutoff, does not alter the relativistic space-time at smaller scales.

Whether $\varkappa$ is a fundamental scale or an auxiliary element of precanonical quantization of fields remains an open question so far. On the one hand, one can show that in free scalar theory $\varkappa$ disappears from the observable characteristics of a quantum field because the spectrum of DW Hamiltonian operator is proportional to $\varkappa$. In interacting scalar theory, powers of $\varkappa$ enter in the perturbative corrections to the spectrum of DW Hamiltonian $\hat{H}$ thus suggesting that $\varkappa$ can be renormalized away by absorbing the expressions with the bare mass and $\varkappa$ in the "observed mass". On the other hand, an estimation of the mass gap in the quantum pure $\mathrm{SU}(2)$ gauge theory [34] derived by precanonical quantization and a naive estimation of the cosmological constant based on the precanonically quantized pure Einstein gravity [35] seem to consistently point to the estimation of the scale of $\varkappa$ at roughly $\sim 10^{2} \mathrm{MeV}$. We hope to clarify the nature of $\varkappa$ in our forthcoming work.

\section{Acknowlegdements:}

I gratefully appreciate the hospitality of the School of Physics and Astronomy of the University of St Andrews, Scotland, and 24/7 availability of its facilities for research. 


\section{References}

1. N. Birrell and P. Davies, Quantum Fields in Curved Space, Cambridge University Press (1982). R.M. Wald, Quantum Field Theory in Curved Spacetime and Black Hole Thermodynamics, University of Chicago Press (1994).

S.A. Fulling, Aspects of Quantum Field Theory in Curved Space-time, Cambridge University Press (1989). L. Parker and D. Toms, Quantum Field Theory in Curved Spacetime, Cambridge University Press (2009). M. Benini, C. Dappiaggi and T.-P. Hack, Quantum field theory on curved backgrounds - a primer, Int. J. Mod. Phys. A 28 (2013) 1330023, arXiv: 1306.0527.

2. I.V. Kanatchikov, Towards the Born-Weyl quantization of fields, Int. J. Theor. Phys. 37 (1998) 333-42, quant-ph/9712058.

3. I.V. Kanatchikov, De Donder-Weyl theory and a hypercomplex extension of quantum mechanics to field theory, Rep. Math. Phys. 43 (1999) 157-70, hep-th/9810165.

4. I.V. Kanatchikov, On quantization of field theories in polymomentum variables, in: Particles, Fields and Gravitation, (Proc. Int. Conf. Łódź, Poland, Apr. 1998) ed. J. Rembieliński, AIP Conf. Proc. vol. 453, p. 356-67, Amer. Inst. Phys., Woodbury (NY) 1998, hep-th/9811016.

5. I.V. Kanatchikov, Geometric (pre)quantization in the polysymplectic approach to field theory, [arXiv:hep-th/0112263].

I.V. Kanatchikov, Covariant geometric prequantization of fields, arXiv : gr-qc/0012038.

6. Th. De Donder, Théorie Invariantive du Calcul des Variations, Gauthier-Villars, Paris (1935).

H. Weyl, Geodesic fields in the calculus of variations, Ann. Math. (2) 36 (1935) 607-29.

H. Rund, The Hamilton-Jacobi Theory in the Calculus of Variations, D. van Nostrand, Toronto (1966).

H. Kastrup, Canonical theories of Lagrangian dynamical systems in physics, Phys. Rep. 101 (1983) 1-167.

7. I.V. Kanatchikov, Rep. Math. Phys. 41 (1998) 49-90, hep-th/9709229.

8. I.V. Kanatchikov, Rep. Math. Phys. 40 (1997) 225-34, hep-th/9710069.

9. F. Hélein and J. Kouneiher, Adv. Theor. Math. Phys. 8 (2004) 735-777, math-ph/0401047.

10. F. Hélein and J. Kouneiher, Adv. Theor. Math. Phys. 8 (2004) 565-601, math-ph/0211046.

11. I.V. Kanatchikov, Novel algebraic structures from the polysymplectic form in field theory, in: GROUP21, Physical Applications and Mathematical Aspects of Geometry, Groups and Algebras, vol. 2, eds. H.-D. Doebner e.a. (World Scientific, Singapore, 1997) 894, arXiv : hep-th/9612255.

12. M. Forger, C. Paufler and H. Römer, Rev. Math. Phys. 15 (2003) 705, math-ph/0202043.

13. I.V. Kanatchikov, Rep. Math. Phys. 46 (2000) 107, hep-th/9911175.

14. I. Kanatchikov, On a generalization of the Dirac bracket in the De Donder-Weyl Hamiltonian formalism, in: Differential Geometry and its Applications, eds. Kowalski O., Krupka D., Krupková O. and Slovák J. (World Scientific, Singapore, 2008) 615, arXiv : 0807.3127.

15. I.V. Kanatchikov, J. Geom. Symmetry Phys. 37 (2015) 43, arXiv: 1501.00480.

16. I.V. Kanatchikov, Ehrenfest theorem in precanonical quantization of fields and gravity, in: The Fourteenth Marcel Grossmann Meeting on Recent Developments in Theoretical and Experimental General Relativity, Astrophysics, and Relativistic Field Theories, Part C, Ed. M. Bianchi, R.T. Jantzen and R. Ruffini (World Scientific, Singapore, 2018) 2828, arXiv:1602.01083 [gr-qc].

17. K. Freese, C. T. Hill and M. T. Mueller, Covariant functional Schrödinger formalism and application to the Hawking effect, Nucl. Phys. B255 (1985) 693-716.

18. S.-Y. Pi, Quantum field theory in flat Robertson-Walker space-time: functional Schrödinger picture, in: Field Theory and Particle Physics, eds. O. Éboli, M. Gomes and A. Santoro (World Scientific, Singapore, 1990) 144-195.

19. D. V. Long and G. M. Shore, Nucl. Phys. B530 (1998) 247-78, hep-th/9605004.

20. D. V. Long and G. M. Shore, Nucl. Phys. B530 (1998) 279-303, gr-qc/9607032.

21. A. Corichi, H. Quevedo, Phys. Rev. D66 (2002) 085025, arXiv: gr-qc/0207088.

22. A. Corichi, J. Cortez and H. Quevedo, Ann. Phys. 113 (2004) 446-78, arXiv : hep-th/0202070.

23. C. Kiefer, Phys. Rev. D44 (1991) 1067-76.

24. B. Hatfield, Quantum Field Theory of Point Particles and Strings, (Reading MA, Addison-Wesley, 1992). 
25. R. Jackiw, Analysis on infinite dimensional manifolds: Schrödinger representation for quantized fields, in: Field Theory and Particle Physics, eds. O. Éboli, M. Gomes and A. Santoro (World Scientific, Singapore 1990) p. 78-143.

26. M.D. Pollock, Acta Phys. Pol. B41 (2010) 1827-46.

27. I.V. Kanatchikov, Phys. Lett. A283 (2001) 25-36, hep-th/0012084.

28. I.V. Kanatchikov, Rep. Math. Phys. 53 (2004) 181-193, hep-th/0301001.

29. I.V. Kanatchikov, Precanonical quantization and the Schrödinger wave functional revisited, Adv. Theor. Math. Phys. 18 (2014) 1249-65, arXiv: 1112.5801 [hep-th].

30. I.V. Kanatchikov, On the precanonical structure of the Schrödinger wave functional, Adv. Theor. Math. Phys. 20 (2016) 1377-96, arXiv: 1312.4518 [hep-th].

31. I.V. Kanatchikov, Schrödinger wave functional in quantum Yang-Mills theory from precanonical quantization, Rep. Math. Phys. 82 (2018) 373, arXiv: 1805.05279 [hep-th].

32. I.V. Kanatchikov, Schrödinger wave functional of a quantum scalar field in static space-times from precanonical quantization, Int. J. Geom. Meth. Math. Phys. 16 (2019) 1950017, arXiv:1810.09968 [hep-th].

33. I.V. Kanatchikov, Precanonical quantum gravity: quantization without the space-time decomposition, Int. J. Theor. Phys. 40 (2001) 1121-49, gr-qc/0012074.

See also: Kanatchikov I., gr-qc/9810076; gr-qc/9912094; gr-qc/0004066.

34. Igor V. Kanatchikov, On the spectrum of DW Hamiltonian of quantum SU(2) gauge field, Int. J. Geom. Meth. Mod. Phys. 14 (2017) 1750123, arXiv:1706.01766 [hep-th].

35. Igor V. Kanatchikov, On the "spin-connection foam" picture of quantum gravity from precanonical quantization, arXiv:1512.09137 [gr-qc];

I.V. Kanatchikov, On precanonical quantization of gravity in spin connection variables, AIP Conf. Proc. 1514 (2012) 73-76, arXiv:1212.6963 [gr-qc];

I.V. Kanatchikov, De Donder-Weyl Hamiltonian formulation and precanonical quantization of vielbein gravity, J. Phys. Conf. Ser. 442, 012041 (2013), arXiv: 1302.2610 [gr-qc];

I.V. Kanatchikov, On precanonical quantization of gravity, Nonlin. Phenom. Complex Sys. (NPCS) 17 (2014), 372-376, arXiv:1407.3101 [gr-qc].

36. R.A. Bertlmann, Anomalies in Quantum Field Theory, (Clarendon Press, Oxford 2000).

37. V. Volterra and B. Hostinský, Opérations Infinitésimales Linéaires, (Gauthier-Villars, Paris, 1938).

38. A. Slavík, Product Integration, its History and Applications, (Matfyzpress, Prague, 2007). http:/ / www.karlin.mff. cuni.cz/ slavik/product/product_integration.pdf.

39. R.D. Gill, Product-integration, in Encyclopedia of Biostatistics, (John Wiley and Sons, 2005), https://doi.org/10. 1002/0470011815.b2a11058.

40. D.J. Saunders, The Geometry of Jet Bundles, (Cambridge University Press, Cambridge, 1989).

41. P.J. Olver, Applications of Lie Groups to Differential Equations, (Springer-Verlag, NY, 1986).

42. J.R. Klauder, Beyond Conventional Quantization, (Cambridge Univ. Press, Cambridge, 2000). See also: J.R. Klauder, Ultralocal scalar field models, Commun. Math. Phys. 18 (1970) 307-318. 